\section{Growing up healthy: How are we doing?}

Although National Osteopathic Medicine (NOM) week (Sept 10-16) just ended, its theme, "Growing up healthy: It's easier when your doctor's a DO," marks an appropriate junction at which to consider just how American children's health is faring and how the osteopathic medical community relates to it. The special focus of this issue of JAOA examines some of the problems physicians encounter in trying to maintain child health and decrease child morbidity and mortality.

In three articles, contributors address the neonatal abnormalities of conjoined twinning, stillbirth, and hypernatremia in breast-fed infants. Another article suggests a protocol that defines the appropriate healthcare standards applicable in a pediatric outpatient teaching clinic. For physicians who are confronted with the possibility that a child has been sexually abused, "Child sexual abuse" outlines a biopsychosocial protocol that will be especially useful. In addition, the Federal Update section contains the latest immunization guidelines, constantly evolving information that no practicing physician should be without.

The problems discussed in these articles represent the multifaceted issues in pediatric medicine today. Just three decades ago, health concerns facing America's youth were vastly different. Back then, poliomyelitis, measles, and infectious enteritis presented major pediatric challenges. Likewise, clinical training focused on the diagnosis and management of acute diseases. Medical intervention could not readily remediate complications associated with prematurity. Surgical correction of complex congenital malformations was limited. Chronic disease was primarily a problem in the adult population. Child health practitioners were not faced with treating drug abuse and sexually transmitted diseases on a regular basis.

The health issues affecting today's pediatric patients reflect remarkable changes in the biomedical and sociocultural milieu. Most clinicians caring for children face decisions and dilemmas unlike anything encountered during training. Chronic disease is now a major issue in child health. In- creased survival of premature neonates with chronic multisystem disease, along with prolonged life for persons with genetically inherited disease, places increased demands on a poorly prepared healthcare system.

Compounding these complexities is the emergence of the HIV infection in the pediatric population during the past 5 years. AIDS now ranks as the ninth leading cause of death among children younger than age 13 years. This disease, once confined to our nation's urban centers, is rapidly expanding even in nonurban areas.

The incidence of HIV infections in infants and children dramatically underscores the inseparable biopsychosocial aspects of the human condition. Disproportionately afflicting the poor and disenfranchised, AIDS is a beacon to our collective conscience that should cause us to reflect on society's status and the physician's role within it.

Other prominent biopsychosocial dilemmas, illustrated by the following sober statistics, deserve similar reflection: One in 4 children lives below the poverty line; 1 in 5 is born to an adolescent parent. One in 6 has no health insurance, and 1 in 7 will not be graduated from high school. Thirtysix percent of pregnant women receive little or no prenatal care; among industrialized nations, America ranks 19th in infant mortality. We spend approximately $12 \%$ of our gross national product on healthcare, yet childhood mortality remains relatively unchanged while adolescent mortality is on the rise.

Healthcare resources are maldistributed. True gains in children's health are not made when huge sums of money are spent on highly technologic interventions that reach only a very small segment of the population. In times of limited financial resources and a general political climate mandating constraint in healthcare expenditures, how should these competitive dollars be spent? Because ambulatory care costs less than inpatient care, more procedures are being performed in an outpatient setting than just a few years ago. Home phototherapy, hyperalimentation, and ventilator care are on the horizon.

Increasingly, capitation or other prepaid managed care delivery models now control medical practice. Hospital stays for healthy newborns, which 

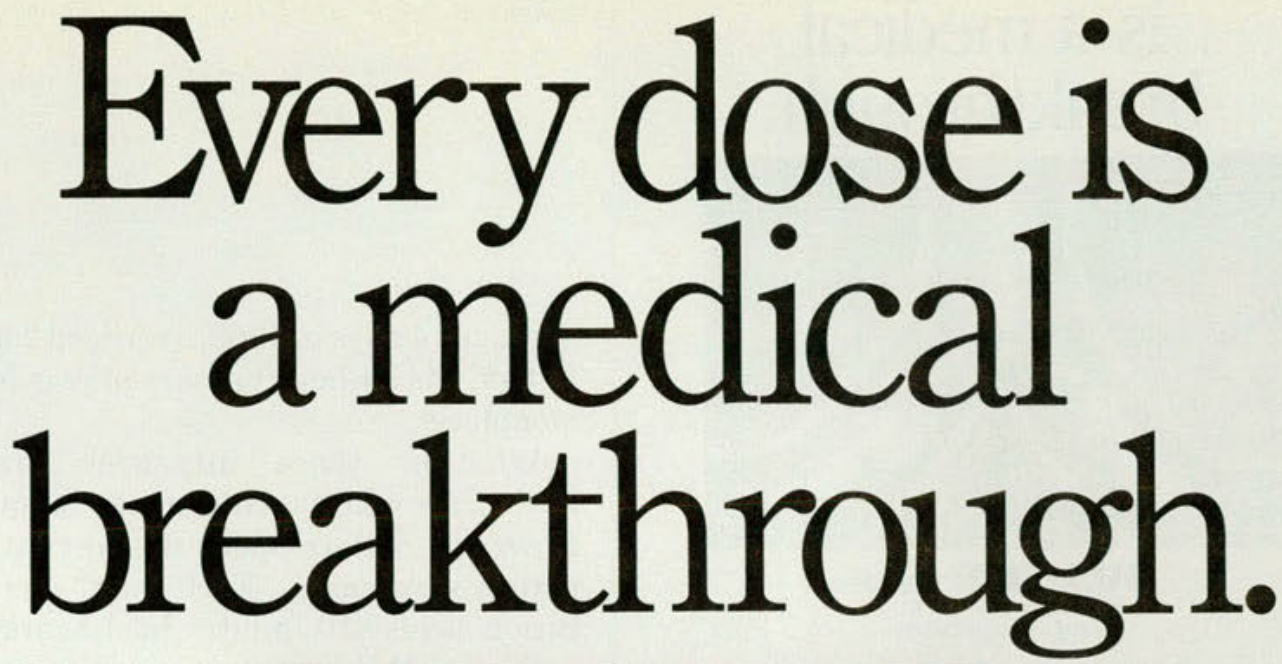

Thick respiratory secretions are the worst part of a cold. Guaifed $^{*}$ can break through this congestion

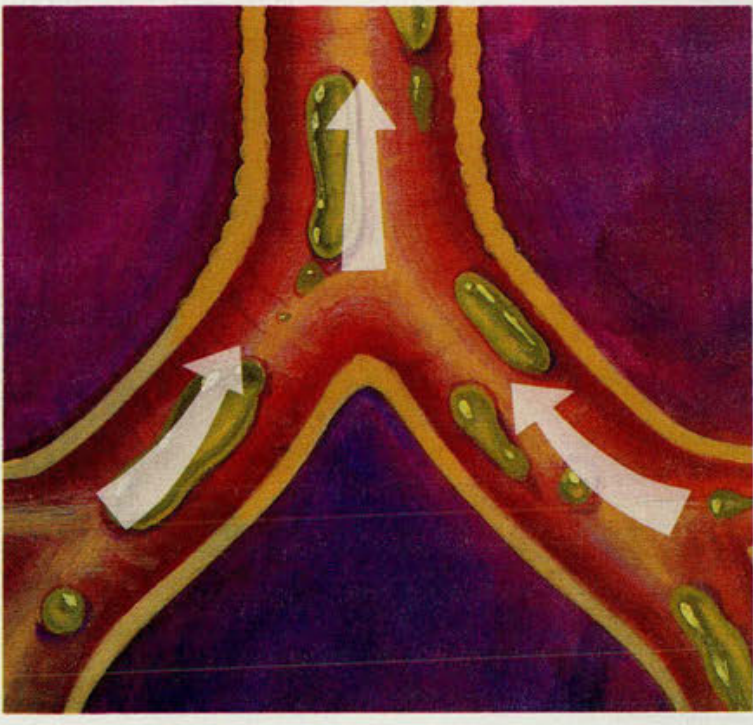

vessels and thereby decreases fluid leakage and swelling of membranes.

There are no anti-

with the first dose. This helps open sinus and bronchial passages for freer breathing.

Guaifenesin, a proven expectorant, helps drain the upper and lower respiratory tract by thinning secretions. It lubricates irritated membranes by increasing mucous flow. It helps remove viscous mucus. Sinus and bronchial drainage is improved. Coughs become more productive and less frequent.

Pseudoephedrine, an effective nasal decongestant, systemically shrinks blood histamines in Guaifed, so the patient is comfortable without feeling drowsy or dried out. Guaifed is designed to release pseudoephedrine $\mathrm{HCl}$ over a prolonged period and to release guaifenesin immediately. It provides a convenient b.i.d. dosage schedule.

Count on Guaifed when you want to break up sinus congestion or a chest cold. Also available as Guaifed-PD ${ }^{\circ}$ Capsules (pseudoephedrine $\mathrm{HCl} 60 \mathrm{mg}$ and guaifenesin $300 \mathrm{mg}$ ).

Please see adjacent page for brief summary of prescribing information.

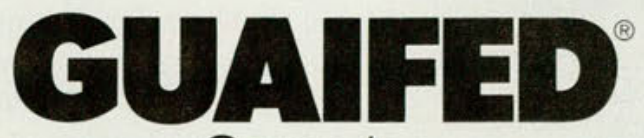

Capsules

(pseudoephedrine $\mathrm{HCl} 120 \mathrm{mg}$ and guaifenesin $250 \mathrm{mg}$ ) 


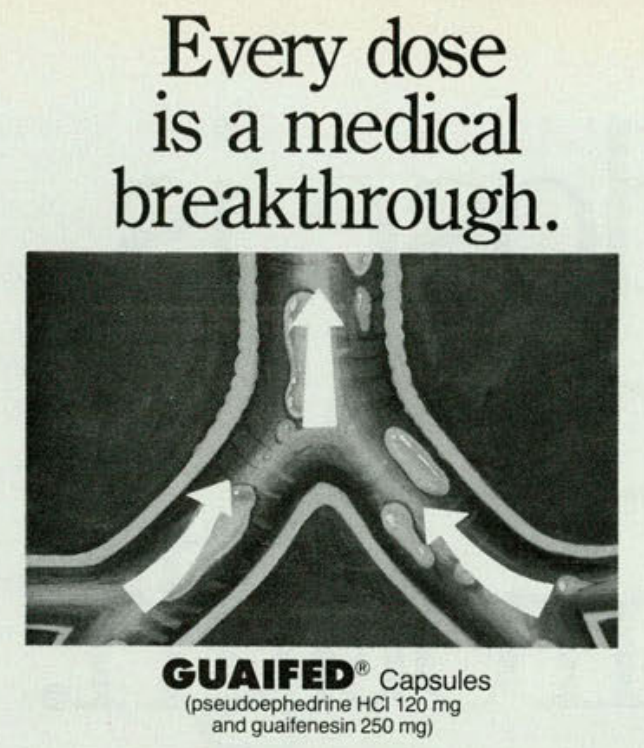

GUAIFED-PD Capsules

(pseudoephedrine $\mathrm{HCl} 60 \mathrm{mg}$ and guaifenesin $300 \mathrm{mg}$ )

Brief Summary

CONTRAINDICATIONS: This product is contraindicated in patients with a known hypersensitivity to any of its ingredients. Also contraindicated in patients with severe hypertension, severe coronary artery disease and patients on MAO inhibitor therapy. Should not be used during preg. nancy or in nursing mothers.

Considerable caution should be exercised in patients with hypertension, diabetes mellitus, ischemic heart disease, hyperthyroidism, increased intraocular pressure and proslatichypertrophy. The elderly (60 years or older) are more the recommended dose, nervousness, diges higher than the recommended dose, nervousness, dizziness or sleep-

PRECAUTIONS: General: Caution should be exercised in patients with high blood pressure, heart disease, diabetes or thyroid disease and in patients who exhibit difficulty in urination due to enlargement of the prostate gland. Check with a physician if symptoms do not improve within 7 days or if accompanied by high fever, rash or persistent headache. Drug Interactions: Do not take this product if you are presently taking a prescription drug for high blood pressure or ently taking a prescription drug for high blood pressure or depression, without first consulting a physician. MAO inhibisympathomimetics. Sympathomimetics may reduce the sympathomimetics. Sympathomimetics may reduce the antinypertensive effects of methyldopa, mecamylamine. chloride may increase the possibility ofcardianedrine hydroin patients presently taking digitalis glycosides. Pregnancy: Pregnancy Category B. It has been shown that pseudoephedrine hydrochloride can cause reduced average weight, length, and rate of skeletal ossification in the

Nursing Mothers: Pseudoephedrine is excreted in breast milk; use by nursing mother is not recommended because of the higher than usual risk of side effects from sympathomimetic amines for infants, especially newborn and prema. ture infants.

Geriatrics: Pseudoephedrine should be used with caution in the elderly because they may be more sensitive to the effects of the sympathomimetics.

WARNINGS: Do not take this product for persistent or chronic cough such as occurs with smoking, asthma, or emphysema, or where cough is accompanied by excessive secretions except under the advice and supervision of a physician. This medication should be taken a few hours prior to bedtime to minimize the possibility of sleeplessness. Take this medication with a glass of water after each dose, to help loosen mucus in the lungs.

ADVERSE REACTIONS: Adverse reactions include nausea, cardiac palpitations, increased irritability or excite ment, headache, dizziness, tachycardia, diarrhea, drowsiness, stomach pain, seizures, slowed heart rate, shortness of breath and/or troubled breathing.

DOSAGE AND ADMINISTRATION: GUAIFED* CAP

SULES Adults and children over 12 years of age: 1 capsule every 12 hours.

GUAIFED-PD* CAPSULES Adults and children over 12 years of age: 1 or 2 capsules every 12 hours. Children 6 to 12 years of age: 1 capsule every 12 hours.

CAUTION: FEDERAL (U.S.A.) LAW PROHIBITS DIS PENSING WITHOUT A PRESCRIPTION.

\section{Distributed by}

\section{Muro}

MURO PHARMACEUTICAL, INC.

TEWKSBURY, MA 01876-9987 editorials

(continued)

averaged 4 days in 1969 , averaged 2.3 days in 1986 . In fact, the 24-hour nursery stay is becoming commonplace.

Whether these measures are improving healthcare while saving money remains to be seen. However, dollars spent on preventive care ultimately save money. Each dollar spent on immunization saves $\$ 10$ in later healthcare costs.

While the struggle continues to provide healthcare to the widest population in the most costeffective manner, we cannot overlook the impact of culture on children's health. Frequently, youngsters are subjected to adult stresses and concerns. They begin to compete for educational achievement as toddlers. Parents, seeking to fulfill their own ego needs, subject their children to developmentally inappropriate academic material.

Tufts University psychologist David Elkind termed this phenomenon "miseducation." Dr Elkind has brought attention to society's tendency to hurry children through childhood. Society places increased pressures on youth to assume adult feelings, roles, and responsibilities. The media, with their portrayal of violence, provocative sexuality, and overwhelming consumerism, are shaping a generation's values and health behaviors.

Against this backdrop, how can we osteopathic physicians help children to grow up healthy? DOs have a long tradition of providing primary, familycentered healthcare. We must continue to deliver excellence in primary care. We must make certain our patients are appropriately immunized and adequately nourished. These fundamental services will go a long way in sustaining strides already made in public health.

We must make children and their parents aware of our availability for psychosocial service. All practitioners, not just pediatricians, must be open to addressing the nonbiologic aspects of patient care. Is the child at risk for abuse? Are parental expectations developmentally appropriate? Is the childor parent(s) - abusing drugs or engaging in other high-risk behaviors? Thoughtful, compassionate, well-intentioned physicians will derive great personal satisfaction from addressing this unmet need. Attention to biopsychosocial unity, in part, distinguishes a great physician from an adequate one.

(continued on page 1129) 
WHEN CONSIDERING LIFESTYLELIMITING EFFECTS OF ANTIHYPERTENSIVE THERAPY...

CAN BETA-BLOCKER THERAPY

LET YOUR PATIENTS

"LIVE AS YOUNG

AS THEY FEEL"? 


\section{IN HYPERTENSION}

\section{PrININIIL (LISINOPRIL/MSD)}

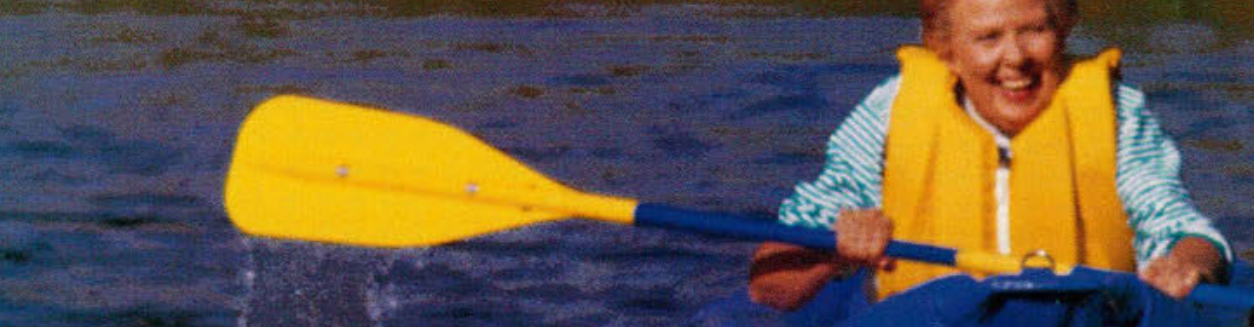

풀
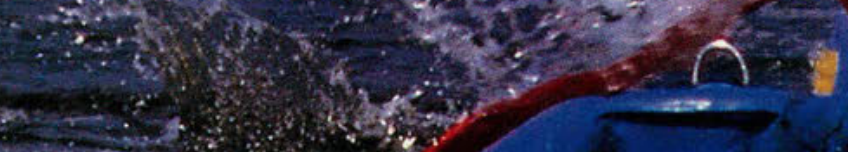

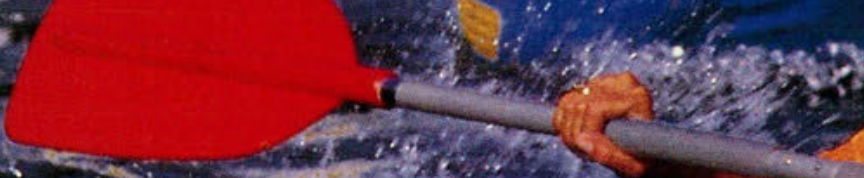

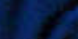




\section{LIVING...AS YOUNG AS THEY FEEL}

\section{COMPARED TO BETA BLOCKERS}

ACE inhibitors such as PRINIVIL ${ }^{\otimes}$ (Lisinopril, MSD) are not characteristically associated with:

reduced cardiac output and heart rate response to exercise

tendency for depression

alterations in lipid profile cold extremities

malaise, drowsiness

\section{COMPARED TO TENORMIN}

A recent clinical study' comparing PRINIVIL and Tenormin demonstrated:

PRINIVIL 20 to $80 \mathrm{mg}$ produced much greater reduction in sitting systolic blood pressure than did Tenormin 50 to $200 \mathrm{mg}(\mathrm{p}<0.01)$, regardless of race. Diastolic reductions were comparable.

Significantly fewer patients receiving PRINIVIL required concomitant thiazide therapy $(\mathrm{p}<0.05)$.*

- PRINIVIL appeared to attenuate diuretic-induced hypokalemia, hyperglycemia, and hyperuricemia.

- Significantly fewer patients receiving PRINIVIL had an increase in serum triglycerides $(p<0.05)$.

1. Bolzano, K. et al.: The antihypertensive effect of lisinopril compared to atenolol in patients with mild to moderate hypertension, J. Cardiovasc. Pharmacol. 9(Suppl. 3):S43-S47, 1987.

*The addition of hydrochlorothiazide caused further reductions $(p<0.01)$ in SSBP/SDBP: PRINIVIL, 16.3/12.5 mm Hg; Tenormin, $18.2 / 9.8 \mathrm{~mm} \mathrm{Hg}$.

NOTE: Tenormin is a registered trademark of ICI Americas Inc.

PRINIVIL is contraindicated in patients who are hypersensitive to this product and in patients with a history of angioedema related to previous treatment with an ACE inhibitor. All patients should be cautioned that excessive perspiration and dehydration may lead to an excessive fall in blood pressure because of reduction in fluid volume. Other causes of volume depletion, such as vomiting or diarrhea, may also lead to a fall in blood pressure. 


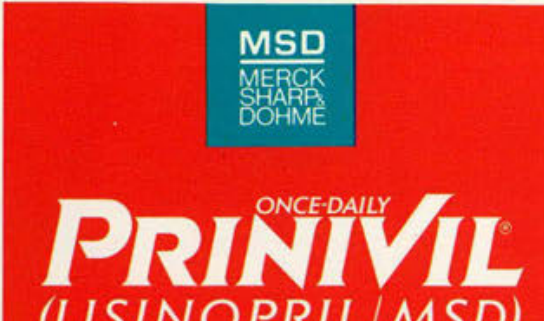

(LISINOPRIL/MSD)

Available in 5-mg, 10-mg, 20-mg, and 40-mg tablets

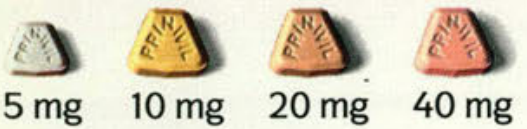

Contraindications:

PRINIVIL* (Lisinopril, MSD) is contraindicated in patients who are hypersensitive to this product and in patients with a history of Warnings:

Angioedema: Angioedema of the face, extremities, lips, tongue glottis, and/or larynx has been reported in patients treated with
ACE inhibitors, including PRINIVIL. In such cases, PRINIVIL should be promptly discontinued and the patient carefully observed until the swelling disappears. In instances where swelling has been the swelling disappears. In instances where swelling has been confined to the face and lips, the condition has generally resolved relieving symptoms. Angloedema associated with laryngeal edema may be fatal. Where there is involvement of the tongue, glottis, or larynx, likely to cause airway obstruction, appropriate therapy, e.g., subcutaneous epinephrine solution $1: 1000(0.3 \mathrm{~mL}$ to $0.5 \mathrm{~mL})$, should be promptly adminis-

Hypotension: Excessive hypotension was rarely seen in uncomplicated hypertensive patients but is a possible consequence of the use of PRINIVIL in salt/volume-depleted persons, such as those treated vigorously with diuretics or patients on dialysis (see PRE-
CAUTIONS, Drug interactions and ADVERSE REACTIONS). In patients with severe congestive heart failure, with or without associated renal insufficiency, excessive hypotension has bee observed and may be associated with oliguria and/or progressive azotemia and rarely with acute renal failure and/or death. Because of the potential fall in blood pressure in these patients, therapy should be started under very close medical supervision. Such patients should be followed closely for the first 2 weeks
treatment and whenever the dose of PRINIVIL and/or diuretic is increased. Similar considerations apply to patients with ischemic heart or cerebrovascular disease in whom an excessive fall in vascular accident.

If hypotension occurs, the patient should be placed in a supine position and, if necessary, receive an intravenous infusion of normal saline. A transient hypotensive response is not a contraindcation to further doses. Which usually can be given without
difficulty once the blood pressure has increased after volume difficulty

Neutropenia/Agranulocytosis: Another ACE inhibitor has been shown to cause agranulocytosis and bone marrow depression. rarely in uncomplicated patients but more frequently in patients with renal impairment, especially if they also have a collagen vas-
cular disease. Available data from clinical trials of PRINIVIL are cular disease. Available data from clinical trials of PRINIVIL are insufficient to show that PRINIVIL does not cause agranulocytosis at similar rates. Periodic monitoring of white blood cell counts in

\section{Precautions:}

General: Impaired Renal Function: As a consequence of inhibitin the renin-angiotensin-aldosterone system, changes in renal function may be anticipated in susceptible individuals. In patients with severe congestive heart failure whose renal function may depend on the activity of the renin-angiotensin-aldosterone system.
treatment with ACE inhibitors, including PRINIVIL, may be associated with oliguria and/or progressive azotemia and rarely with acute renal failure and/or death

In hypertensive patients with unilateral or bilateral renal artery stenosis, increases in blood urea nitrogen and serum creatinine may occur. Experience with another ACE inhibitor suggests that PRINIVIL and/or diuretic therapy. In such patients renal function should be monitored during the first few weeks of therapy.

Some hypertensive patients with no apparent preexisting renal vascular disease have developed increases in blood urea nitrogen and serum creatinine, usually minor and transient, especially when PRINIVIL has been given concomitantly with a diuretic. This ment. Dosage reduction o

Evaluation of the hypertensive patient should always include assessment of renal function (see DOSACE AND ADMINISTRATION)

Hyperkalemia: In clinical trials, hyperkalemia (serum potassium patients and $4.0 \%$ of patients with congestive heart failure. In most cases, these were isolated values which resolved despite of therapy in approximately $0.1 \%$ of hypertensive patients. Ris factors for the development of hyperkalemia include renal insuffciency, diabetes mellitus, and the concomitant use of potassiumsparing diuretics, potassium supplements, and/or potassium containing sait substitutes, which should be with PRINIVIL (see Drug Interactions)

ing anesthesia with agents that oroduce hypotension, PRINIVIL may block angiotensin II formation secondary to compensatory renin release. If hypotension occurs and is considered to be due to Information for Patients:

Angioedema: Angioedema, including laryngeal edema, may Occur, especially following the first dose of PRINIVIL' (Lisinopril,
MSD). Patients should be so advised and told to report immediately any signs or symptoms suggesting angioedema (swelling of face, extremities, eyes, lips, tongue, difficulty in swallowing or breathing) and to take no more drug until they have consulted with the prescribing physician

sion: Patients should be cautioned to report lightheadedness, especially during the first few days of therapy. If actual syncope occurs, patients should be told to dis-
continue the drug until they have consulted with the prescribing physician.

All patients should be cautioned that excessive perspiration and dehydration may lead to an excessive fall in blood pressure depletion such as vomiting or diarrhea may also lead to a fall in depletion such as vomiting or diarrhea may also lead to a fall in
blood pressure; patients should be advised to consult with their physician.

Hyperkalemia: Patients should be told not to use salt substitutes containing potassium without consulting their physician.

Veutropenia: Patients should be told to report promptly any indication of infection (e.g., sore throat, fever) which may be a sign of

treated with PRINIy other drugs, certain advice to patients being to aid in the safe and effective use of this medication. It is not a disclosure of all possible adverse or intended effects.

Drug Interactions:

Hypotension: Patients on Diuretic Therapy: Patients on diuretics and especially those in whom diuretic therapy was recently instituted, may occasionally experience an excessive reduction of blood pressure after initiation of therapy with PRINIVIL. The pos-
sibility of hypotensive effects with PRINIVIL can be minimized by sibility of hypotensive effects with PRINIVIL can be minimized by
either discontinuing the diuretic or increasing the salt intake prior to initiation of treatment with PRINIVIL. If it is necessary to conto initiation of treatment with PRINetic, initiate therapy with PRININIL at a dose of $5 \mathrm{mg}$ daily, and provide close medical supervision after the initial dose for at least 2 hours and until blood pressure has stabilized for at ADMINISTRATION) When a diuretic is added to the therapy of a patient receiving PRINIVIL, an additional antihypertensive effect patient receiving PRINIVIL, an additional antihypertensive effect Is usually observed. Studies with ACE inhibitors in combination
with diuretics indicate that the dose of the ACE inhibitor can be reduced when it is given with a diuretic (see DOSAGE AND ADMINISTRATION,

Indomethacin: In a study in 36 patients with mild to moderate hypertension where the antihypertensive effects of PRINIVIL
alone were compared to PRINIVIL given concomitantly with indoalone were compared to PRINIVIL given concomitantly with indomethacin, the use of indomethacin was associated with a reduced
effect, although the difference between the two regimens was not significant.

Other Agents: PRINIVIL has been used concomitantly with nitrates and/or digoxin without evidence of clinically significant adverse interactions. No clinically important pharmacokinetic
interactions occurred when PRINIVIL was used concomitantly interactions occurred when PRINIVL was used concomitantly
with propranolol or hydrochlorothiazide. The presence of food in with propranolol or hydrochlorothiazide. The presence of
the stomach does not alter the bioavailability of PRINIVIL. Agents Increasing Serum Potassium: PRINIVIL attenuates potas-
sium loss caused by thiazide-type diuretics. Use of PRINIVIL with Sium loss caused by thiazide-type diuretics. Use of PRINIVIL with
potassium-sparing diuretics (e.g., spironolactone, triamterene, or amiloride), potassium supplements, or potassium-containing salt substitutes may lead to significant increases in serum potassium. Therefore, if concomitant use of these agents is indicated because of demonstrated hypokalemia, they should be used with caution and with frequent monitoring of serum potassium.
Pregnancy Category C: Lisinopril, at doses up to $1 \mathrm{mg} / \mathrm{kg} / \mathrm{day}$, was not teratogenic when given throughout the organogenic period in saline-supplemented rabbits. Saline supplementation (physiologic saline in place of tap water) was used to eliminate maternotoxic effects and enable evaluation of the teratogenic potential at the highest possible dosage level. The rabbit has been shown to be extremely sensitive to ACE inhibitors (captopril and enalapril)
with maternal and fetotoxic effects apparent at or below the recwith maternal and fetotoxic effects apparent at or below the recdemmended therapeutic dosage levels in man. Fetotoxicity was resorptions at an oral dose of lisinopril at $1 \mathrm{mg} / \mathrm{kg} / \mathrm{day}$ and by an increased incidence of incomplete ossification at the lowest dose tested $(0.1 \mathrm{mg} / \mathrm{kg} /$ day). A single intravenous dose of $15 \mathrm{mg} / \mathrm{kg}$ of lisinopril administered to pregnant rabbits on gest
21 , or 26 resulted in $88 \%$ to $100 \%$ fetal death.

There are no adequate and well-controlled studies of lisinopril in pregnant women. However, data are available that show drugs of this class cross the human placenta. Because the risk of fetal toxicity with the use of ACE inhibitors has not been clearly defined. benefit justifies the potential risk to the fetus.

Postmarketing experience with all ACE inhibitors thus far suggests the following with regard to pregnancy outcome. Inadvertent reported to affect fetal outcome adversely. Fetal exposure during the second and third trimesters of pregnancy has been associated with fetal and neonatal morbidity and mortality.

When ACE inhibitors are used during the later stages of pregnancy, there have been reports of hypotension and decreased renal perfusion in the newborn. Oligohydramnios in the mother has also been reported. presumably representing decreased tors should be closely observed for hypotension, oliguria, and hyperkalemia. If oliguria occurs, attention should be directed toward support of blood pressure and renal perfusion with the administration of fluids and pressors as appropriate. Problems associated with prematurity such as patent ductus arteriosus but it is not clear whether they are related to ACE inhibition, maternal hypertension, or the underlying prematurity.

Nursing Mothers: It is not known whether this drug is secreted in human milk. Because many drugs are secreted in human milk,
caution should be exercised when PRINIVIL is given to a nursing Pediatric Use: Safety and effectiveness in children have not been Adverse Reactions:

PRINIVIL has been found to be generally well tolerated in controlled clinical trials involving 2.003 patients and subjects.
The most frequent clinical adverse experiences in controlled trials with monotherapy with PRINIVIL* (Lisinopril, MSD) were: dizziupper respiratory symptoms $(3.0 \%)$, and cough (2.9\%), all of which were more frequent than in placebo-treated patients. For the most part, adverse experiences were mild and transient in the most part, adverse experiences were mild and transient in
nature. Discontinuation of therapy was required in $6 \%$ of patients. In clinical trials, the overall frequency of adverse experiences could not be related to total daily dosage within the recommended therapeutic dosage range.

Other adverse experiences occurring in greater than $1 \%$ of
patients and subjects treated with monotherapy with PRINIVIL in controlled clinical trials were: nausea (2.3\%), hypotension chest pain (1.3\%), vomiting (1.3\%), and dyspnea (1.1\%). For information on adverse experiences occurring in greater than $1 \%$ of patients and subjects treated with PRINIVIL plus hydrochloro-
thiazide in controlled clinical trials, see Prescribing Information. Clinical adverse experiences occurring in $0.3 \%$ to $1 \%$ of patients treated with monotherapy with PRINIVIL in the controlled trials and rarer, serious, possibly drug-related events reported in uncontrolled studies or marketing experience included: Body as 0 Whole. Chest discomfort, fever, flushing. Cardiovascular: Angina cardia, peripheral edema, palpitation. Digestive: Abdominal pain anorexia constipation, flatulence. Metabolism: Gout. Mus culoskeletal: Joint pain, shoulder pain. Nervous System/Psychiatric: Depression, somnolence, insomnia, stroke. Respiratory guria, progressive azotemia, acute renal failure. Other: Blurred vision, pruritus, urinary tract infection, vasculitis of the legs ing PRINIVIL (0.1\%). Angioedema associated with laryngeal edema may be fatal. If angioedema of the face, extremities, lips. tongue, glottis, and/or larynx occurs, treatment with PRINIVIL should be discontinued and appropriate therapy instituted immediately (see WARNINCS). Hypotension: In hypertensive patients. hypotension occurred in $1.2 \%$ and syncope occurred in $0.1 \%$ of of therapy in $0.5 \%$ of hypertensive patients (see WARNINCS). In patients with congestive heart failure, hypotension occurred in $5 \%$ and syncope occurred in $1 \%$ of patients. These adverse expepatients. patients.

Serum Electrolytes: Hyperkalemia (see PRECAUTIONS).

Creatinine, Blood Urea Nitrogen: Minor increases in blood urea therapy, were observed in about $2 \%$ of patients with essential hypertension treated with PRINIVIL alone. Increases were more patients with renal artery stenosis (see PRECAUTIONS). Revers were observed in approximately $9.1 \%$ of patients with congestive heart failure on concomitant diuretic therapy. Frequently, these abnormalities resolved when the dosage of the diuretic was decreased

Hemoglobin and Hematocrit: Small decreases in hemoglobin and hematocrit (mean decreases of approximately $0.4 \mathrm{~g} \%$ and $1.3 \mathrm{vol}$ $\%$, respectively) occurred frequently in patients treated with some other cause of anemia. In clinical trials, less than $0.1 \%$ of patients discontinued therapy due to anemia.

Other (Causal Relationship Unknown): Rarely, elevations of liver

nzymes and/or serum bilirubin have occurred.
Overall, $2.0 \%$ of patients discontinued therapy due to laboratory adverse experiences, principally elevations in blood urea nitroge Dosage and Administration

hitial Therapy: In patients with uncomplicated essential hypertension not on diuretic therapy, the recommended initial dose is
$10 \mathrm{mg}$ once a day. Dosage should be adjusted according to blood pressure response. The usual dosage range is 20 to $40 \mathrm{mg}$ per day administered in a single daily dose. The antihypertensive effect the administered dose. but most commonly with a dose of $10 \mathrm{mg}$ daily. This can be evaluated by measuring blood pressure just prior to dosing to determine whether satisfactory control is being maintained for 24 hours. If it is not, an increase in dose should be considered. Doses up to $80 \mathrm{mg}$ have been used but do not appear to give a greater effect. If blood pressure is not controlled with Hydrochlorothiazide $12.5 \mathrm{mg}$ has been shown to provide an additive effect. After the addition of a diuretic, it may be possible to reduce the dose of PRINIVIL

Diuretic-Ireated Patients: In hypertensive patients who are currently being treated with a diuretic, symptomatic hypotension diuretic should be discontinued. if possible, for 2 to 3 days before beginning therapy with PRINIVIL to reduce the likelihood of hypoadjusted according to blood pressure response. If the patient's therapy may be resumed as described above.

If the diuretic cannot be discontinued, an initial dose of $5 \mathrm{mg}$

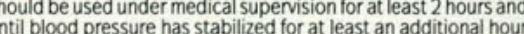
see WARNINGS and PRECAUTIONS. Drug Interactions. Concomitant administration of PRINIVIL with potassium supplements, potassium salt substitutes, or potassium-sparing diuretics Use in Elderily: In general, blood pressure response and adverse experiences were similar in younger and older patients given simi-

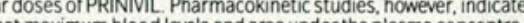
tion time curve (AUC) are and area under the plasma concentraadjustments should be made with particular caution.

Dosage Adjustment in Renal Impairment: The usual dose of RIN $3 \mathrm{mg} / \mathrm{dL}$ ). For patients with creatinine clearance $\geq 10 \mathrm{~mL} / \mathrm{min}$ and once daily. For patients with creatinine clearance $<10 \mathrm{~mL} / \mathrm{min}$ usually on hemodialysis), the recommended initial dose is is controlled or to a maximum of $40 \mathrm{mg}$ daily.

For more detailed information, consult your MSD Representative 
Promoting children's health should be the primary focus of any practice. While DRGs are a hospital reality, implementation of a resource-based relative value scale for physician services seems imminent. This proposed redistribution of medical moneys, being developed by Harvard University through a study commissioned by the Health Care Financing Administration, may be a boost to primary care practitioners and others who provide time-intensive, cognitive-type services. However, procedural biotechnology experts stand to lose monetarily if such changes occur.

While the debate surrounding physician reimbursement continues, we must maintain our efforts to enhance child healthcare on the local, regional, and national levels. Participation in organizations such as school boards, PTAs, and youth groups affords us the opportunity to serve our communities. In addition to such "grass roots" activism, membership in osteopathic professional organizations provides us with an avenue to effect change. The American College of Osteopathic Pediatricians (ACOP) is one organization whose primary focus is to foster and stimulate improvement in all areas of child health and well-being. The ACOP, which marks its 50th year in 1990 , welcomes all licensed physicians interested in children's health as members.

As of now, NOM week may be over, but our commitment to meet the challenge of children growing up healthy should know no time constraints.

RONALD V. MARINO, DO

Special focus editor

Dr Marino is Director of Ambulatory and Behavioral Pediatrics at Winthrop-University Hospital, Mineola, NY, and a member of the JAOA Editorial Advisory Board.

\section{AOA joins fight against sexually transmitted diseases}

While the AIDS epidemic commands the attention of the medical community and the media, the incidence of other sexually transmitted diseases (STDs) quietly festers. The incidence of Chlamydia, genital herpes, gonorrhea, genital warts, and syphilis is increasing at an alarming rate in the United States: According to the Centers for Disease Control (CDC), the number of syphilis cases increased from approximately 70,000 in 1986 to 103,000 in 1988. The incidence of drug-resistant strains of gonorrhea rose from 22,000 in 1987 to 31,000 cases in 1988. Each year, approximately 3 to 4 million Chlamydia infections occur. Likewise, 750,000 new cases of genital warts and 500,000 cases of symptomatic genital herpes are diagnosed annually.

These statistics reveal only part of the problem. The real crux of the issue can be attributed, in part, to public misinformation. A survey commissioned by the Burroughs Wellcome Co found that of the polled 632 adults between the ages of 18 and 49 , $62 \%$ never questioned a sexual partner or potential partner about having an STD. Twenty-seven percent believed that STDs can be contracted from a toilet seat or other surface, and $12 \%$ thought that a person cannot contract STDs through a single sexual encounter with a familiar companion.

This survey represents part of an educational campaign designed to encourage sexually active individuals to conduct a regular genital self-examination (GSE ${ }^{(\pi)}$ ). Recognizing the importance of this campaign, the American Osteopathic Association, along with the American College of General Practitioners in Osteopathic Medicine and Surgery (ACGPOMS); the American Academy of Dermatology; the American Academy of Family Physicians; and the American Social Health Association, has joined the Burroughs Wellcome Co in its education efforts.

To shed light on this current health crisis, the sponsors have produced a pamphlet, "Genital SelfExamination GSE Guide." The pamphlet describes, in easy-to-understand language, the causes and symptoms of the five major STDs previously mentioned. It details a step-by-step self-examination procedure in which men and women can check themselves for possible STD signs and symptoms. Patients who think they may have an STD are encouraged to seek medical attention, not only for diagnosis and treatment, but for advice on how to prevent the spread of the disease. Physicians may

(continued on page 1132 ) 